\title{
High Detection Efficiency and Time Resolution Integrated-Passive-Quenched Single-Photon Avalanche Diodes
}

\author{
Fabio Acerbi, Member, IEEE, Massimo Cazzanelli, Alessandro Ferri, Alberto Gola, Member, IEEE, \\ Lorenzo Pavesi, Senior Member, IEEE, Nicola Zorzi, and Claudio Piemonte, Member, IEEE
}

\begin{abstract}
We present a new silicon integrated-passive-quenched single-photon avalanche diode, fabricated at FBK. Unlike common SPADs, they feature the quenching resistor lithographically fabricated close to the detector and they also have a special topmetallization layout which allows a better signal extraction. We characterized the performance of devices with different active area, layout and junction technology, particularly focusing on the timing jitter. We studied the effect of the metallization layout on timing jitter, the differences between two types of technologies and we also compared measurements performed with blue light and nearinfrared light. These devices showed a remarkable timing jitter, close to $20 \mathrm{ps}$ full-width at half-maximum.
\end{abstract}

Index Terms-Photodetectors, photon timing, picosecond, single photon, SiPM, SPAD, TCSPC, time resolution.

\section{INTRODUCTION}

$\mathbf{S}$ INGLE-photon detectors are employed in many applications that require to measure faint light signals with high detection efficiency and very good time resolution. Among the others, single-photon avalanche diodes (SPADs) offer the typical advantages of solid state devices, like ruggedness, integrability, high detection efficiency, low power consumption, insensitivity to magnetic fields, and they are often a good choice for practical applications thanks to their compactness and ease of use.

A SPAD is basically a p-n junction reverse biased above its breakdown voltage by an amount called "excess bias". The electric field within the active area is so high that even a single charge carrier can trigger a self-sustaining impact ionization process eventually leading to a macroscopic current, easily detectable by front-end electronics, and which can be quenched by an external circuit. The leading-edge of the current marks precisely the photon arrival time, in case of photogeneration. Instead, a thermally or tunnel generated carrier gives a "dark count".

Manuscript received January 31, 2014; revised June 11, 2014; accepted July 17,2014 . This work was supported in part by the European Community Seventh Framework Programme (EU FP7) Project SUB Nanosecond Leverage In PET/MR ImAging (SUBLIMA) under Grant 241711.

F. Acerbi, A. Ferri, A. Gola, N. Zorzi, and C. Piemonte are with the Center for Materials and Microsystems, Fondazione Bruno Kessler, I-38123 Trento, Italy (e-mail: acerbi@fbk.eu; aleferri@fbk.eu; gola@fbk.eu; zorzi@fbk.eu; piemonte@fbk.eu).

M. Cazzanelli and L. Pavesi are with the Nanoscience Laboratory, Department of Physics, University of Trento, I-38123 Trento, Italy (e-mail: massimo.cazzanelli@unitn.it; lorenzo.pavesi@unitn.it).

Color versions of one or more of the figures in this paper are available online at http://ieeexplore.ieee.org.

Digital Object Identifier 10.1109/JSTQE.2014.2341580
Passive quenching is the simplest quenching method [1]: a high-value resistor $\left(\mathrm{R}_{\mathrm{q}}\right)$ is connected in series to the SPAD and when the macroscopic avalanche current rises, the resistor lowers the SPAD bias (discharging the diode capacitance), eventually quenching it. The higher the overall diode and parasitic capacitances (seen by the SPAD), the bigger is the avalanche charge produced [1]. On one hand, a bigger charge gives a higher signal but, on the other hand, it gives a higher after-pulsing probability. In order to limit the charge down to reasonable values, integrated passive quenching (IPQ) (resistor lithographically fabricated close to the SPAD) was introduced. This leads also to a shorter recharge time of the detector.

Active quenching [1] allows to promptly restore the SPAD bias and to precisely control the hold-off time (tens of nanoseconds [2]-[4]): they are typically preferable for "single-pixel" SPAD or array of SPADs for timing applications [5]-[7]. However, thanks to its simplicity and small area occupation, passive quenching, and particularly the iPQ, has been employed for analog silicon photomultipliers (SiPMs), where one of the main figure of merits is the pixel fill factor (FF), and also in a new kind of InGaAs/InP SPAD with "negative feedback" [8] to reduce the avalanche charge, and thus the afterpulsing (the main bottleneck of this technology) [9], [10].

In this paper we analyze and characterize new silicon integrated-passively-quenched single-photon avalanche diodes (iPQ-SPADs), fabricated at FBK (Trento, Italy), exploiting the same technology employed for SiPMs. We tested SPADs with circular and squared active areas, with different layouts and from n-on-p [11] or p-on-n [12] junction-type technologies. We particularly focus on timing performance. These single-photon detectors have very good detection efficiency and, despite the relatively small output signal, they proved to have very good timing resolution. At room temperature we measured about $20 \mathrm{ps}$ full-width at half-maximum (FWHM), with blue light, and $25 \mathrm{ps}$ with near-infrared light (for circular SPADs with 20- $\mu \mathrm{m}$ diameter), which are comparable with the best reported values for silicon SPADs [13]-[15].

\section{Device Structures}

We tested iPQ-SPADs from two different technologies (n-on-p junction [11] and p-on-n junction [12]), with circular and square active areas. Table I summarizes the characteristics of tested devices. In both types of circular SPADs a metal ring is placed all around the active area and covering the n-implant, 
TABLE I

LIST OF MEASURED DEVICES

\begin{tabular}{lccc}
\hline \hline Technology & Active area layout & Diameter/side $(\mu \mathrm{m})$ & Metallization sub-version \\
\hline n-on-p & circular & 20 & Type-A \\
n-on-p & circular & 20 & Type-B \\
n-on-p & circular & 100 & Type-B \\
n-on-p & square & 50 & Type-A \\
p-on-n & circular & 20 & \\
\hline \hline
\end{tabular}

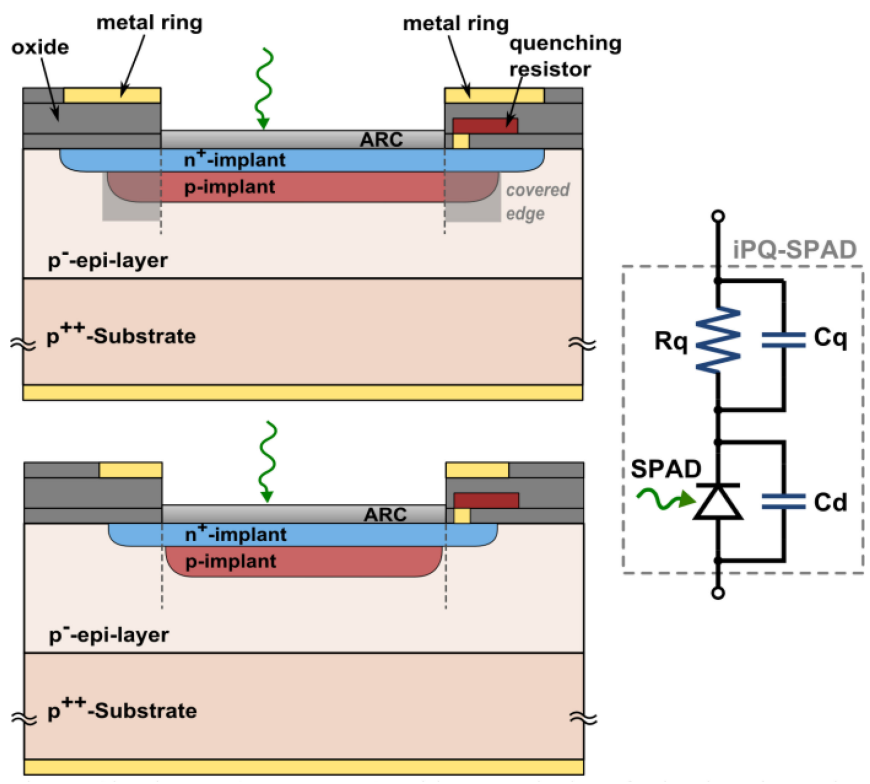

Fig. 1. Circular n-p SPAD type-A, with covered edge of p-implant, i.e., active area (top) and type-B, uncovered edge (bottom), and equivalent circuit (Rq is the quenching resistor, $\mathrm{Cq}$ is the "quenching capacitance", $\mathrm{Cd}$ is the diode capacitance). Metal ring is connected directly to output pad.

whereas the square SPAD does not have it. Moreover, for the circular structures, we designed two versions: one with the metal ring overlapping part of the active area ("type-A") whilst the other one outside it ("type-B"), as shown in Fig. 1. The diameter of the photosensitive region, i.e., uncovered active area, is the same in both circular versions, thus, in type-A, p-type enrichment region is slightly bigger.

This metal ring is electrically connected to the output pad increasing the so-called "quenching capacitance" (with reference to the model in [16] shown in Fig. 1). This configuration allows a better extraction of the avalanche current and produces a high and fast peak component in the output signal. As a first approximation, timing jitter is proportional to the baseline-fluctuation of the signal and inversely proportional to its rising-edge slope (around the threshold level) [17], [18]. Therefore, commonly, in timing measurements, the higher is the signal amplitude, the higher is the signal first-derivative at the low-voltage threshold used, and the lower is the timing jitter.

\section{Detection EFficiency AND Dark Count Rate}

We characterized the photon detection efficiency (PDE) of $n$ on-p and of p-on-n technologies employing $1 \times 1 \mathrm{~mm}^{2}$ SiPMs,

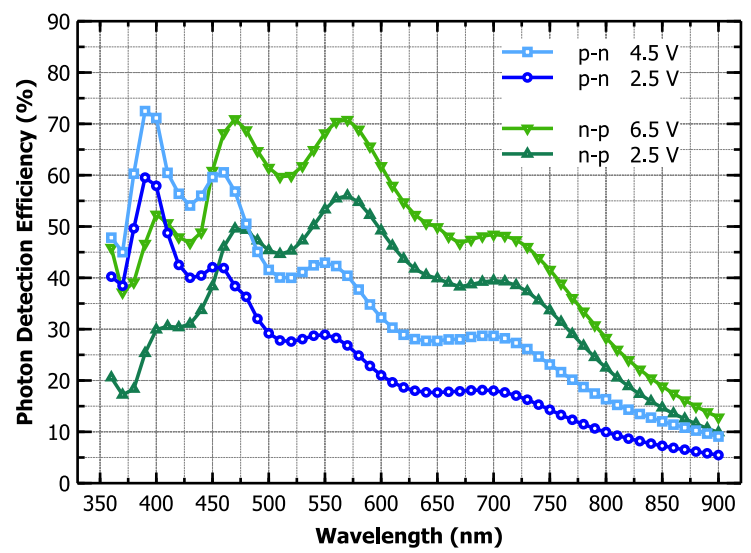

Fig. 2. PDE of SPADs made in n-on-p and p-on-n technologies: values have been obtained by measurements on $1 \times 1 \mathrm{~mm}^{2}$ SiPMs and divided by their FF

with $50 \mu \mathrm{m}$ side cells. The setup used is composed by a broadspectrum light source, optical neutral filters and a monochromator; a calibrated reference detector has been positioned in place of the DUT to characterize the system and then primary count rate of the SiPM has been measured (afterpulsing and crosstalk have been excluded), with and without light, for dark count rate subtraction. The values obtained were divided by the $\mathrm{FF}$ (FF $\sim 45 \%$ for both technologies) to obtain the single-device efficiency and the results are reported in Fig. 2.

As expected, p-on-n technology has enhanced PDE in the blue-light region, thanks to the electron-triggered avalanches for the carriers generated close to the silicon surface. The triggering probability at $390 \mathrm{~nm}$ reaches its maximum at about $4.5 \mathrm{~V}$ of excess bias $(\sim 30 \mathrm{~V}$ reverse bias) giving a PDE higher than $70 \%$. We cross-checked with other measurements the PDE of the circular SPADs employed in this work and the PDE results were always comparable to the ones obtained with SiPM (divided by $\mathrm{FF}$ ), confirming the remarkable detection efficiency.

Concerning the n-on-p technology, the maximum PDE is in the green region. It reaches values of about $57 \%$ at $2.5 \mathrm{~V}$ of excess bias ( $29 \mathrm{~V}$ reverse bias) and higher than $70 \%$ at $6.5 \mathrm{~V}$.

We measured also the noise of these detectors, employing the characterization procedure described in [19], which is based on avalanche-pulse inter-time plot to distinguish primary dark counts and afterpulsing. The $20-\mu \mathrm{m}$ circular SPAD made in $\mathrm{p}$ on-n technology has a very low noise: the dark count rate is 40 counts per second (cps) at $20^{\circ} \mathrm{C}$, at $3 \mathrm{~V}$ of excess bias, and of $70 \mathrm{cps}$ at $6 \mathrm{~V}$ (in line with the SiPM noise when normalized to SPAD active area). Conversely, despite the new n-on-p device production runs have also very low dark count rate, the particular production run employed for timing measurements in this paper has a relatively high noise, of more than one order of magnitude higher than p-on-n technology. It was the first production with both SiPM and single-cell on the same wafer. This high noise produces effects also on timing response at room temperature, as explained in the next section.

\section{TIMING JITTER}

The timing jitter of a SPAD is defined as the spread in time between photon absorption and pulse detection by timing 


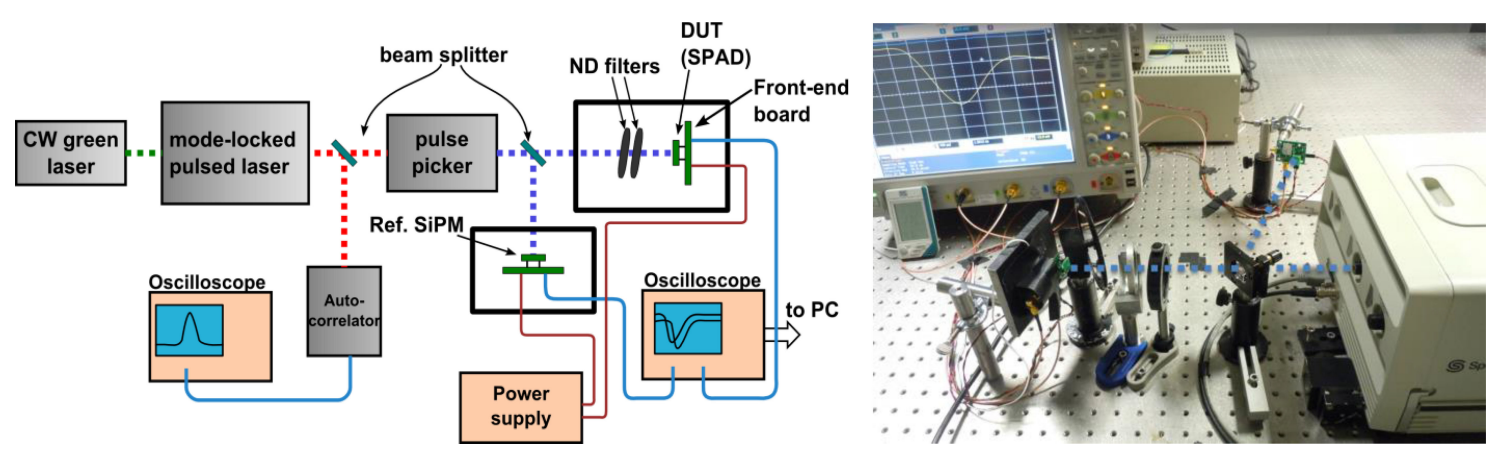

Fig. 3. Schematic representation and photograph of the setup employed for timing jitter measurements.

electronics (in our case, the overcoming of a threshold by the amplified output signal). Measurements were performed illuminating the device with narrow laser pulses (attenuated to single photon level) and evaluating the arrival-time dispersion of electric signal from the front-end circuit.

\section{A. Setup}

As shown in Fig. 3, we employed a mode-locked Ti:Sapphire laser (from the Nanoscience Lab., Department of Physics, University of Trento), pumped by a continuous-wave green laser, providing red-pulses (tunable in wavelength around $800 \mathrm{~nm}$ ) with a temporal width of about 2 ps FWHM and a repetition rate of $82 \mathrm{MHz}$. Since this frequency is higher or comparable with the typical recharge time of these detectors (typically few hundreds on nanoseconds), we also employed an additional pulse-picker, synchronized with the laser, which periodically select one optical pulse over $N$ (we selected an output pulse frequency of few tens of kilohertz). This block can also integrate a second harmonic generator, allowing us to measure the timing jitter in the blue-wavelength region, too. The output light spot has a diameter of about $2 \mathrm{~mm}$.

The optical signal was attenuated to the single-photon level by neutral-density filters (detection rate on the SPAD was below $1 \%$ of the laser pulse rate) and sent to the DUT. Then, the amplified detector signal (we employed an AD8000 amplifier) and the reference trigger signal were fed to the $1 \mathrm{GHz}$ bandwidth, high sampling-rate (20 GSample/sec) Agilent oscilloscope, which acquired, digitalized and sent data to the PC. A LABVIEW program performed the arrival-time statistics, thus the timing jitter measurements.

It must be considered that a very precise synchronization signal is mandatory for this jitter measurement, locked with the optical pulses. We performed a preliminary system characterization exploiting the electrical SYNC OUT from the laser, obtaining a minimum resolution of tens of picoseconds. To have a better trigger signal, we decided to exploit the optical laser pulse itself, splitting it into two paths: the first non-attenuated is sent to a "Reference" SiPM, connected to a front-end circuit equal to the one employed for the DUT; the second, attenuated is sent to the DUT. The reference was illuminated by thousands of photons per pulse and operated at a fixed low excess bias. To quantify the system resolution capability (i.e., contributions

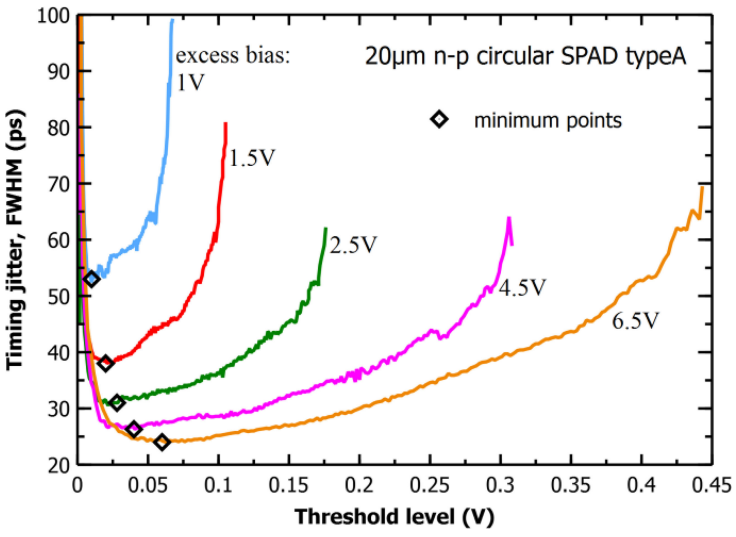

Fig. 4. Timing jitter FWHM, at a wavelength of $400 \mathrm{~nm}$, at different excess bias, as a function of threshold voltage. Minimum values are marked: note that there is not an "optimal" threshold value for all excess biases.

from the detectors, front-end circuitry and oscilloscope acquisition and digitalization) we measured the timing jitter between the output signals of two identical SiPMs (both optical paths without filters), obtaining a coincidence time resolution of less than 10 ps FWHM. This means a trigger jitter of less than 7 ps FWHM (subtracting in quadrature), which is sufficiently low for our measurements (laser width gives a small contribution).

Measurements were performed at room temperature $\left(\sim 25{ }^{\circ} \mathrm{C}\right)$, with detectors placed in optically-isolated blackboxes to reduce their dark count rate to the minimum.

\section{B. On-Line Analysis}

Acquired waveforms were analyzed with a custom LABVIEW program. Compared with a traditional system based on time-correlated single-photon counting board, this on-line analysis not only allows implementing different types of real-time filtering, but it also allows extracting timing-response curves at different thresholds (typically hundreds of levels) at the same time, without having to adjust the setup. Fig. 4 shows acquired timing jitter values for $20 \mu \mathrm{m}$ n-on-p circular type-A SPAD illuminated with $400-\mathrm{nm}$ pulses. With low thresholds the jitter is high, due to the electronic noise and baseline fluctuations. Then there is a minimum and after that the curves rise and diverge when the threshold reaches the value of the pulse amplitude. 


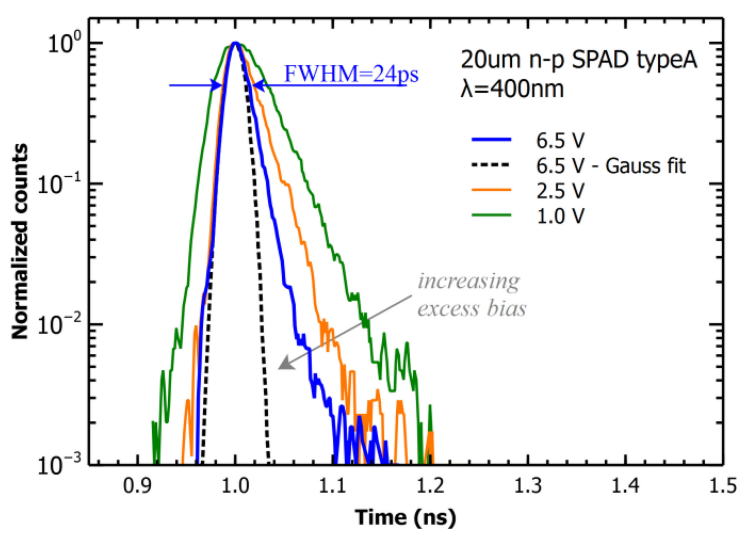

Fig. 5. Timing response of n-over-p-junction 20- $\mu$ m circular SPAD type-A at a wavelength of $400 \mathrm{~nm}$, at different excess biases. Dashed curve is the Gaussian fit on the $6.5 \mathrm{~V}$ curve.

Note that the rising of the jitter in the low-threshold region is more important with high excess biases, due to the higher baseline fluctuation induced by the higher DCR. Therefore, the threshold value giving the minimum time-jitter is not the same for each bias and an "optimal" value for all conditions does not exist. In our measurements, we always took the minimum as the timing jitter value.

\section{Performance of n-on-p SPADs at $400 \mathrm{~nm}$}

The timing response distribution of the n-on-p junction 20- $\mu$ m circular type-A SPAD, at $400-\mathrm{nm}$ is shown in Fig. 5. At this wavelength, almost all incoming photons are absorbed within the depleted region (absorption length $\sim 0.1 \mu \mathrm{m}$ ). As an example with $2.5 \mathrm{~V}$ of excess bias we measured a timing jitter FWHM of $\sim 30 \mathrm{ps}$, while it reduces to about $\sim 24 \mathrm{ps}$ at higher excess bias. The timing response is very sharp, even at low bias. It approaches quite well a Gaussian distribution (see dashed line). The small tail could be given by the photons absorbed in the superficial non-depleted region (diffusing and triggering the avalanche with delay) or, as explained below, could be generated by previous dark counts.

We characterized this SPAD also at $425 \mathrm{~nm}$. Fig. 6 summarizes the timing jitter as a function of the excess bias. There is no evident difference between the two wavelengths. The dashed line represent FWHM of the Gaussian fit, performed considering mainly the left side of the histogram: this is about $5 \mathrm{ps}$ less than the measured data for most of the bias values, confirming the quasi-Gaussian shape. These are very good results for passively-quenched SPADs, and they also are among the best results ever reported [13]-[15] for "standard" silicon-SPADs.

The low timing jitter can be attributed to both: i) an optimized front-end board, with reduced electrical noise and good signal amplification, ii) the metal ring covering the edge of the n-p junction area. Concerning the first point, we characterized timing jitter with several front-end topologies (e.g., trans-impedance, series resistance with positive amplification, etc.) and gains: we saw that with AD8000 amplifier chip, the single-amplifier solution in a trans-impedance configuration produces the higher

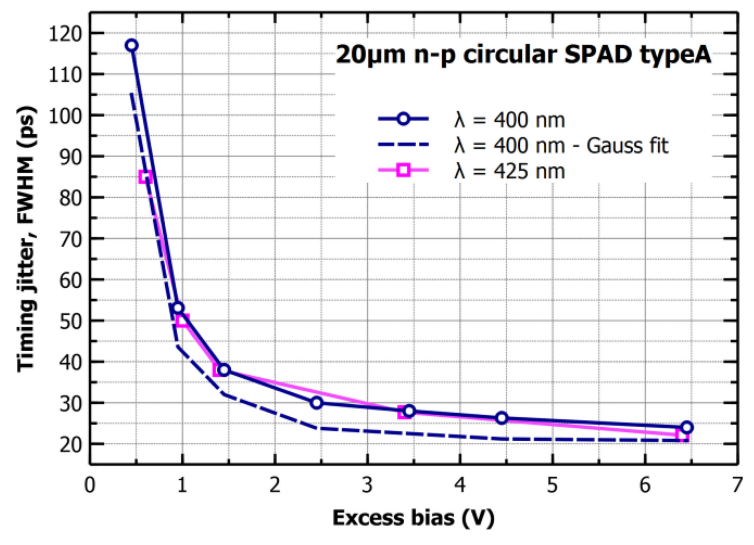

Fig. 6. Timing jitter FWHM as a function of excess bias for p-over-n $20 \mu \mathrm{m}$ SPAD measured at 400 and $425 \mathrm{~nm}$. Dashed line represent FWHM of the Gaussian fit on 400-nm curves.

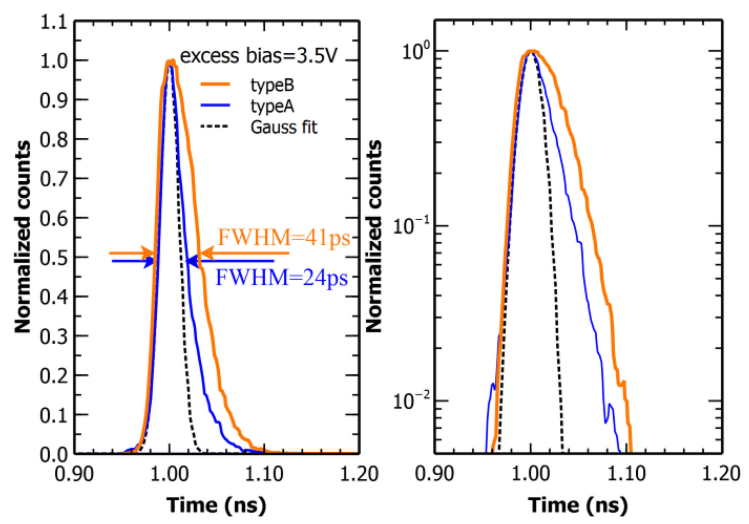

Fig. 7. Comparison between timing response of $\mathrm{n}-\mathrm{on}-\mathrm{p}-\mathrm{junction} 20-\mu \mathrm{m}$ circular SPAD type-A and type-B (with linear and log vertical scales).

bandwidth, thus the steeper signal and the best results. Concerning the second point, the metal ring has two effects: i) it increases the quenching capacitance $\left(C_{q}\right)$, giving a good extraction of the signal with a prompt peak, and ii) it covers the edge of the detector active area (other than the shallow implant), which generally have a slightly lower electric field with respect to the central region. Previous works [15] report that, excluding the very first part of avalanche build-up, the center-triggered avalanche grows faster than the border-triggered one, giving better timing jitter. The border of active area has usually a slightly lower electric field and worse timing performance.

In order to quantitatively check how this metal-shielding improves timing performance, we characterized another $20-\mu \mathrm{m}$ circular SPAD (type-B), with uncovered active-area edge. The shape of its timing histogram is very similar to the type-A one but with a bigger slow component after the main peak (as shown in Fig. 7). It can be noted that the left side of the histograms overlaps very well, and the Gaussian fit is very similar between type-A and type-B. The larger slow component is mainly due to the light absorption in the edge region where electric field has a lower value. Indeed, the overall SPAD timing histogram is the superposition of many "local" contributions, each one with a peak position and width that depends on the local electric field. 


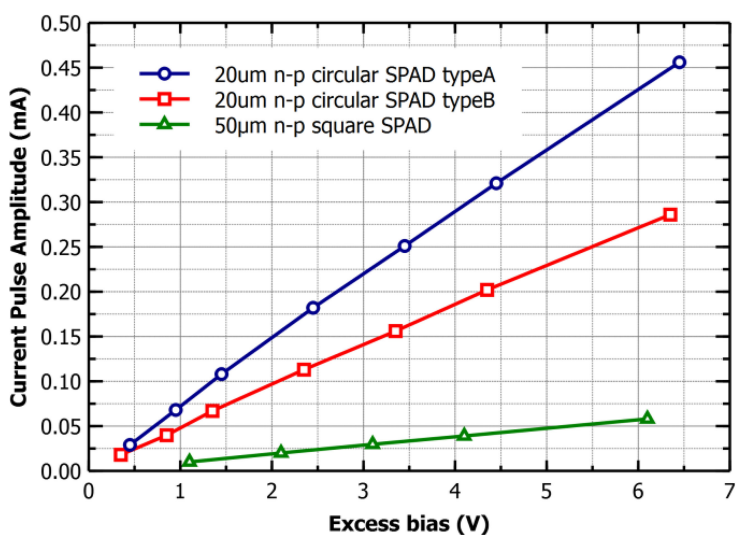

Fig. 8. Average current pulse amplitude as a function of excess bias, for $20 \mu \mathrm{m}$ circular SPADs and square SPAD.

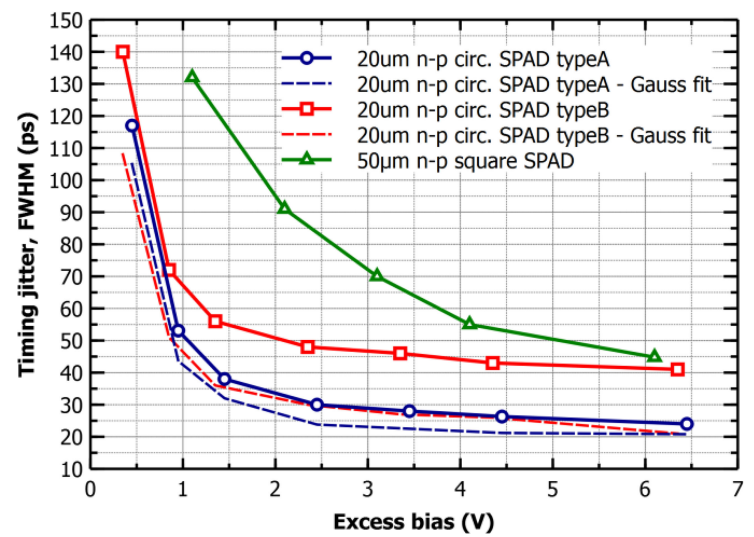

Fig. 9. Timing jitter (FWHM) as a function of the excess bias for p-on-n $20 \mu \mathrm{m}$ SPAD type-A and type-B, measured at $400 \mathrm{~nm}$ (the FWHM of the Gaussian fits are also shown, which are quite similar to each other) and of $50 \mu \mathrm{m}$ square SPAD, measured at $425 \mathrm{~nm}$.

The lower the field, the more delayed and wider is the peak of the "local" response.

We also characterized the timing performance of another SPAD, with $50-\mu \mathrm{m}$-side squared active area, which is very similar to the single cell composing a SiPM [11]. It does not have the metallization covering the shallow implant, thus the output signal is lower. A comparison of the current pulse amplitude of the three tested SPADs is shown in Fig. 8: the highest is the circular SPAD type-A, as expected, since: i) its metal ring covers a bigger portion of the $n-p$ junction area, ii) the effective $\mathrm{n}-\mathrm{p}$ junction area is bigger than in type-B. The type-B has amplitude about $40 \%$ less than type-A. The square SPAD has much lower amplitude, about $15 \%$ than type-A circular one, despite the bigger area, indicating that the metal ring covering shallow implant is very helpful in avalanche signal extraction.

The effect of amplitude on timing jitter is shown in Fig. 9. Square SPAD has a higher jitter, with respect to circular devices, particularly at low excess biases where the signal amplitude is low, and it gets better while its bias is increased, reaching values similar to SPAD type-B. Circular SPADs seems to have a fixed difference due to edge shielding. The effects produced

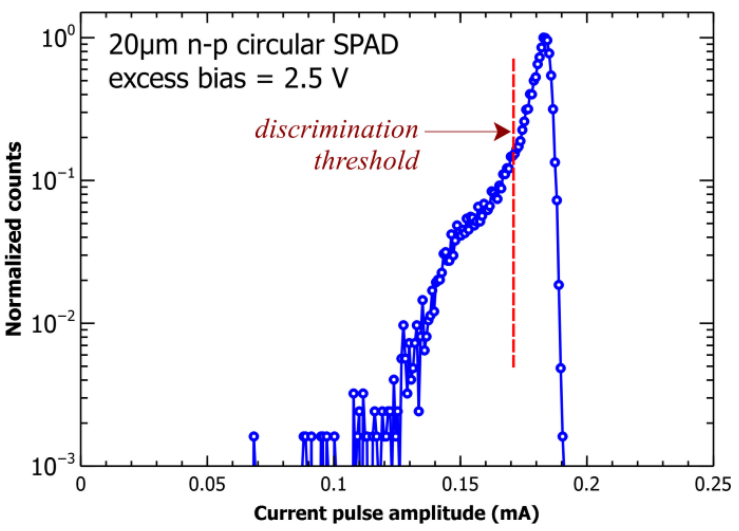

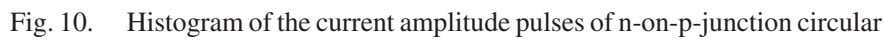
type-A SPAD, at $2.5 \mathrm{~V}$ of excess bias.

by the slightly lower amplitude of type-B (respect to type-A) are probably covered by the edge effect.

\section{Pulse Amplitude Discrimination}

Another aspect that we considered is the effect of dark counts on the timing response. In common applications, SPAD are cooled to decrease DCR [3], [4]. In our measurements, the devices were operated at room temperature (due to setup limitations). Exploiting the on-line analysis capability of our setup, we histogrammed also the pulse amplitude distribution. In the ideal case, a Gaussian shape is expected. In reality, as shown in Fig. 10, there is also a tail produced by pulses with lower amplitude. They are generated by laser-triggered events happening just after a dark event, i.e., where the detector bias is still not fully recovered. The higher the DCR, the more significant this tail is.

Hence, to study SPAD timing performance at a more "physical" level, i.e., excluding the effect of dark counts and considering build-up and quenching statistics, we characterized again the timing jitter but considering only the pulses with amplitude above the discrimination threshold shown in Fig. 10. The timing response histogram of circular SPAD type-A, at 2.5 and $6.5 \mathrm{~V}$ of excess bias, are shown in Fig. 11, in comparison with the ones acquired without amplitude discrimination. The effect of the lower-amplitude pulses is an increased tail after the main peak. Pulses with lower amplitude cross the threshold level with a delay with respect to the case of full amplitude.

Using the same procedure for the other SPADs, we obtained the timing jitters reported in Fig. 12. The improvement in FWHM for circular SPADs is about 4 ps, bringing the minimum timing jitter to $\sim 20$ ps FWHM. For the square device, the difference is higher at low excess bias (i.e., about 25 ps), due to the larger active area, (thus higher DCR) and reduces at higher values where likely the effect of the uncovered edge become visible and limits the improvements when amplitude is discriminated.

Using the amplitude discrimination we also measured another circular device with active area diameter of $100 \mu \mathrm{m}$ and uncovered edge. As shown Fig. 12, it has an excess bias dependence 

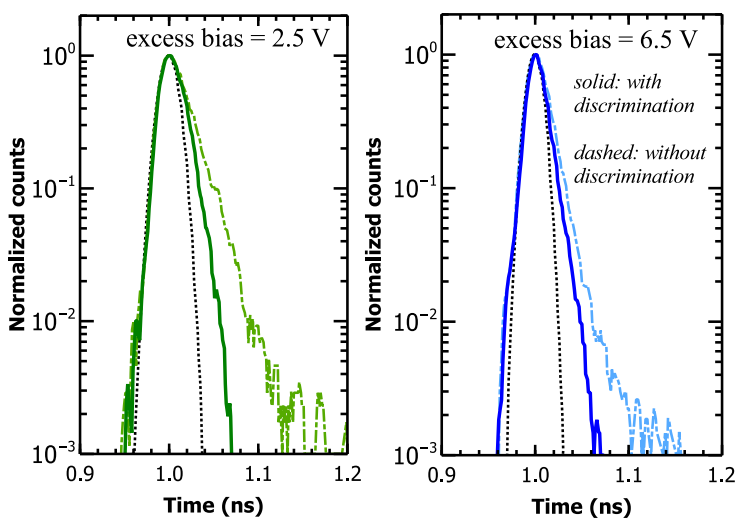

Fig. 11. Timing response of n-on-p-junction circular SPAD type-A with and without the minimum pulse amplitude discrimination, with $2.5 \mathrm{~V}$ of excess bias (left) and $6.5 \mathrm{~V}$ (right). The difference is in the tail, whereas the rising part and the Gaussian fit are the same.

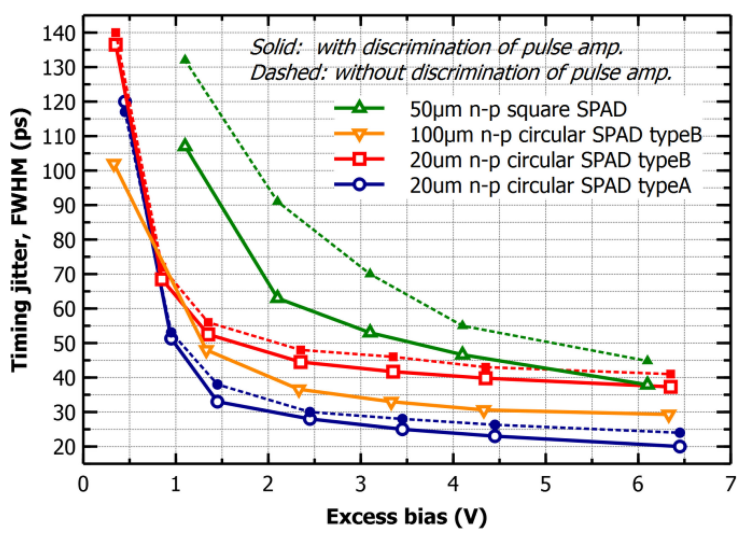

Fig. 12. Timing jitter FWHM of circular and square SPADs, with and without the minimum pulse amplitude discrimination.

very similar to other circular devices. At very low bias its FWHM is better, thanks to the larger current pulse amplitude. Moreover, it is interesting to notice that its timing jitter is lower than the one of the $20 \mu \mathrm{m}$ type-B SPAD. This is because, despite the bigger active area (giving more spread in avalanche build-up statistics), the ratio of the center-area to the edge-area is higher than in $20 \mu \mathrm{m}$ SPADs, thus the effect of uncovered border is reduced and the jitter is more similar to type-A SPAD.

Therefore, excluding $20 \mu \mathrm{m}$ type-B SPAD, where the effect of uncovered active-area edge dominates time resolution, and considering only $20 \mu \mathrm{m}$ type-A SPAD, $100 \mu \mathrm{m}$ type-B SPAD and $50 \mu \mathrm{m}$ square SPAD, we can summarize as follows: i) the big area worsen the timing resolution of about $10 \mathrm{ps}$, ii) with a square area and without any metal ring timing resolution is another $10 \mathrm{ps}$ worse than for the big-area circular device.

\section{E. $n-p$ SPADs at $850 \mathrm{~nm}$}

Removing the second harmonic generation stage, we performed timing jitter measurements in the near-infrared wavelength region, at $850 \mathrm{~nm}$ (absorption length $\sim 18 \mu \mathrm{m}$ ). In this condition, a large part of the incoming photons is absorbed in

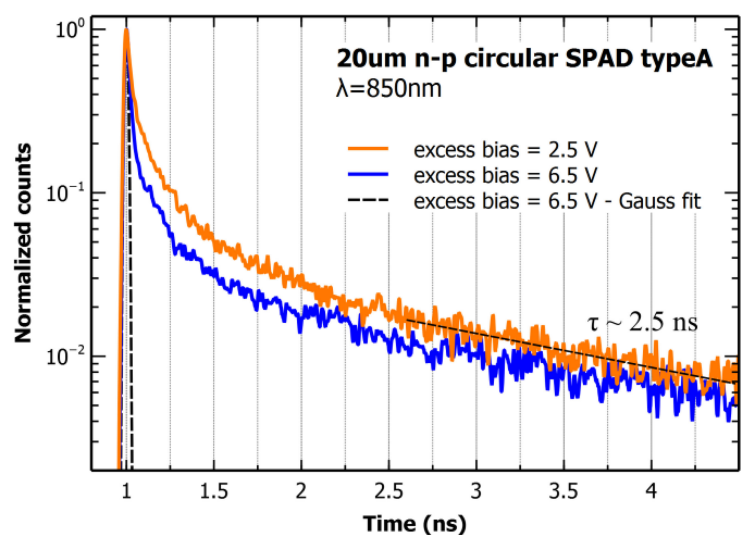

Fig. 13. Timing response of the n-on-p circular type-A SPAD at $850 \mathrm{~nm}$, for two excess bias values.

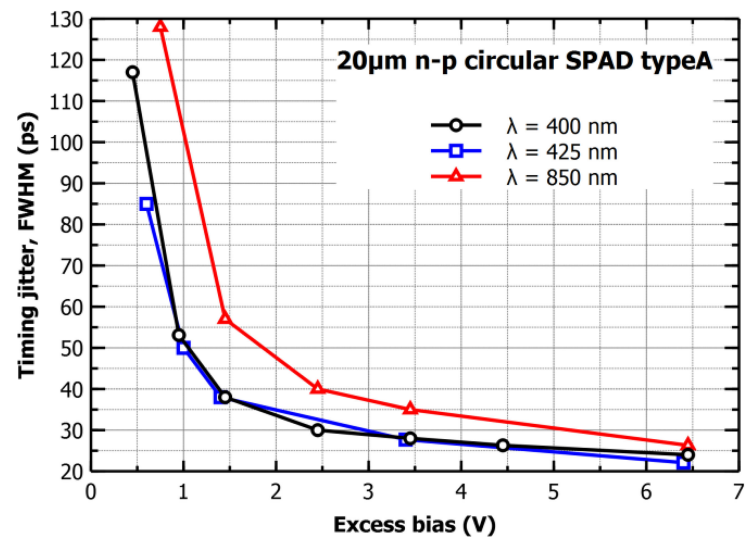

Fig. 14. Timing jitter FWHM of $20 \mu$ m circular SPAD type-A at 400, 425, and $850 \mathrm{~nm}$, as a function of excess bias.

the neutral zone below the depleted region, which is composed by non-depleted epitaxial layer and the substrate. As shown in Fig. 13, the timing histogram shows a relevant tail with a time constant $\sim 2.5 \mathrm{~ns}$. This behavior is given by the diffusion of carriers generated in the neutral regions towards the depleted one. The amplitude of the tail, normalized to the amplitude of the main peak, lowers when the excess bias is increased because the width of the depleted region grows (more rapidly at excess bias around $3 \div 5 \mathrm{~V}$ and then more slowly above $5 \mathrm{~V}$ ). This effect increases the timing jitter FWHM of the detector. In principle it can be removed with more complex epitaxial structure (e.g., [20]).

Fig. 14 shows the timing jitter at the three different wavelengths employed: at $850 \mathrm{~nm}$, at low excess bias, the jitter is about 20 ps FWHM higher than in the blue-wavelength region, whereas the difference reduces to only few picoseconds at high excess biases, where the tail amplitude is relatively low. At $6 \mathrm{~V}$ of excess bias the timing jitter is still lower than $30 \mathrm{ps}$ (note that we did not apply pulse amplitude discrimination in measurement at $850 \mathrm{~nm}$ ).

\section{F. Performance of $p-n S P A D$}

A different SPAD technology is made with a shallow-p and deep-n implant. Photons absorbed superficially initiate the 


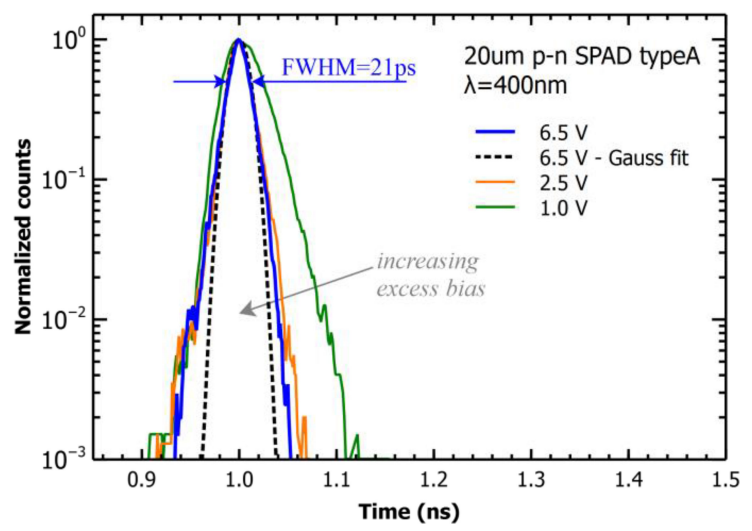

Fig. 15. Timing response of p-on-n-junction 20- $\mu \mathrm{m}$ circular SPAD type-A at $400 \mathrm{~nm}$, at different excess biases.

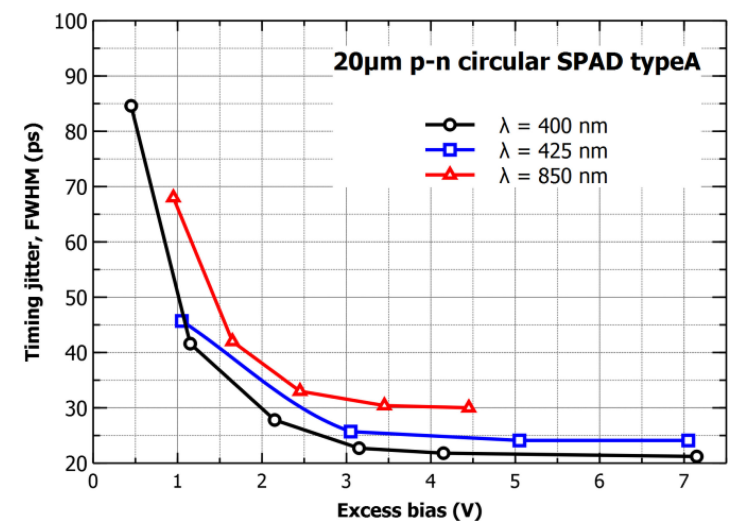

Fig. 16. Timing jitter FWHM of $20 \mu \mathrm{m}$ p-n circular SPAD type-A at 400, 425 , and $850 \mathrm{~nm}$, as a function of excess bias.

avalanche multiplication process with electrons, whereas photons absorbed deep into the bulk initiate the avalanche with holes.

We characterized timing performances of a $20-\mu \mathrm{m}$ circular type-A SPAD, at $400 \mathrm{~nm}$, and the results are reported in Fig. 15, for different excess biases. Timing response distributions are almost Gaussian. The tail is significantly smaller than the corresponding SPAD made with n-p junction mainly because of the considerably lower DCR of this technology. The measured timing jitter FWHM is slightly better than n-p SPADs: as shown in Fig. 16, at $2.1 \mathrm{~V}$ of excess bias it is lower than $28 \mathrm{ps}$, whereas at $6.5 \mathrm{~V}$ of excess bias is around 20 ps without any pulse discrimination.

We also measured the timing response with the other two wavelengths used for the previous detectors. At $425 \mathrm{~nm}$ timing jitter is slightly higher than at $400 \mathrm{~nm}, \sim 3 \mathrm{ps}$. This could be because, at the lower wavelength, most of the photons are absorbed in the shallow-implant region, before the electric-field peak, thus avalanches are triggered by electron whereas, at $425 \mathrm{~nm}$, part of photons is absorbed at the other side of the electric-field peak, triggering the avalanche with holes, and giving a slightly higher spread in the buildup process.

With 850-nm light pulses the worsening of jitter (with respect to the blue-ligh case) is similar to that of $n-p$ devices: tens of

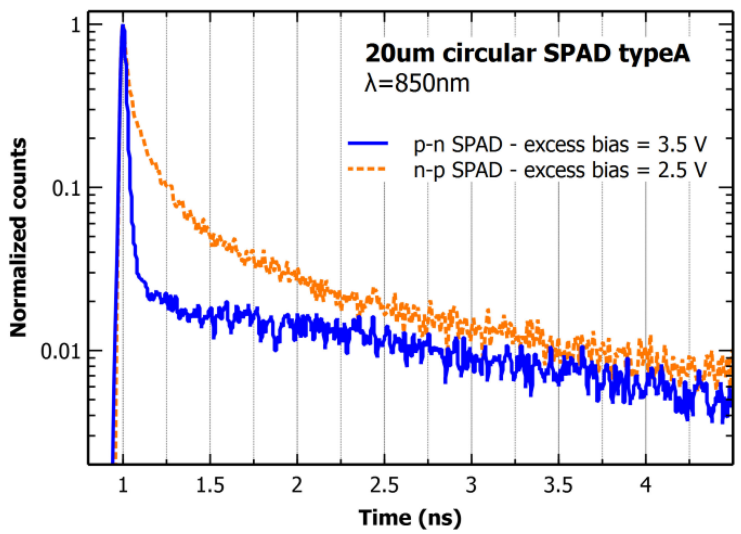

Fig. 17. Timing response of p-on-n circular SPAD type-A at $850 \mathrm{~nm}$, in comparison with n-on-p response.

picoseconds at low excess biases, which reduces to $\sim 10 \mathrm{ps}$ at about $2.5 \mathrm{~V}$. Timing jitter is $30 \mathrm{ps}$ FWHM at about $4 \mathrm{~V}$ of excess bias.

In the timing histogram of p-n SPAD, shown in Fig. 17, the tail amplitude is small (about one fiftieth of the main peak amplitude) and the main peak is well defined, particularly when compared with the n-p SPAD response (dashed line). This could be thanks to a triggering probability of the photons absorbed in the bulk that is lower than for photons absorbed in the upper region, whereas the situation is the opposite in $n-p$ devices.

\section{CONCLUSION}

In this work we characterized iPQ-SPADs, fabricated at FBK, with the same technology employed for SiPMs. Quenching resistor is micro-fabricated close to the detector. We characterized devices with circular and square active area from n-on-p or p-on-n junction technologies.

Circular SPADs have a metal ring around the active area: this increases the amplitude of the signal extracted from the detector, improving timing performance (as confirmed by the comparison between square SPAD without top metal and circular SPAD with metal). Moreover, when this ring covers the edge of active area, where electric field is lower, we measure an improvement of about 20 ps in timing jitter FWHM.

We also measured a circular SPAD with $100 \mu$ m diameter: despite the bigger area, the ratio between central-area and borderarea is greater than the previous devices, giving a timing jitter that is halfway between the covered and uncovered $20 \mu \mathrm{m}$ SPADs, confirming the effect of the border.

The results shown in this paper are very promising, among the best ever reported for silicon-SPADs. Circular devices with $20-\mu \mathrm{m}$ diameter show a jitter between 20 and 24 ps FWHM, with blue light, for both n-on-p and p-on-n technologies at room temperature.

At $850 \mathrm{~nm}$ a tail with $\sim 2.5 \mathrm{~ns}$ time constant appears after the main peak, but the resulting FWHM is still low, between $\sim 25$ and $30 \mathrm{ps}$. 


\section{REFERENCES}

[1] S. Cova, M. Ghioni, A. L. Lacaita, C. Samori, and F. Zappa, "Avalanche photodiodes and quenching circuits for single photon detection," Appl. Opt., vol. 35, no. 12, pp. 1956-1976, Apr. 1996.

[2] A. Gulinatti, I. Rech, P. Maccagnani, M. Ghioni, and S. Cova, "Largearea avalanche diodes for picosecond time-correlated photon counting," in Proc. 35th Eur. Solid-State Device Res. Conf., Grenoble, France, 2005, pp. $355-358$.

[3] Micro Photon Devices, PDM datasheet. (2014). [Online]. Available: http://www.micro-photon-devices.com/

[4] F. Zappa, S. Tisa, A. Tosi, and S. Cova, "Principles and features of singlephoton avalanche diode arrays," Sens., Actuators A, Phys., vol. 140, no. 1, pp. 103-112, 2007.

[5] C. Cammi, F. Panzeri, A. Gulinatti, I. Rech, and M. Ghioni, "Custom single-photon avalanche diode with integrated front-end for parallel photon timing applications," Rev. Sci. Instrum., vol. 83, no. 033104, pp. 033104-1-033104-9, 2012.

[6] D. Stoppa, D. Mosconi, L. Pancheri, and L. Gonzo, "Single-photon avalanche diode CMOS sensor for time-resolved fluorescence measurements," IEEE Sensors J., vol. 9, no. 9, pp. 1084-1090, Sep. 2009.

[7] C. Niclass, C. Favi, T. Kluter, M. Gersbach, and E. Charbon, "A $128 \times$ 128 single-photon image sensor with column-level 10-bit time-to-digital converter array," IEEE J. Solid-State Circuits, vol. 43, no. 12, pp. $2977-$ 2989, Dec. 2008.

[8] M. A. Itzler, X. Jiang, B. M. Onat, and K. Slomkowski, "Progress in selfquenching InP-based single photon detectors," Proc. SPIE, vol. 7608 , pp. 760829-1-760829-13, Feb. 2010.

[9] M. A. Itzler, X. Jiang, M. Entwistle, K. Slomkowski, A. Tosi, F. Acerbi, F. Zappa, and S. Cova, "Advances in InGaAsP-based avalanche diode single photon detectors," J. Modern Opt., vol. 58, no. 3/4, pp. 174-200, 2011.

[10] M. Liu, C. Hu, X. Bai, X. Guo, J. C. Campbell, Z. Pan, and M. M. Tashima, "High-performance InGaAs/InP single-photon avalanche photodiode," IEEE J. Sel. Topics Quantum Electron., vol. 13, no. 4, pp. 887-894, Aug. 2007.

[11] N. Serra, A. Ferri, A. Gola, T. Pro, A. Tarolli, N. Zorzi, and C. Piemonte, "Characterization of new FBK SiPM technology for visible light detection," J. Instrum., vol. 8, p. 03019, Mar. 2013.

[12] T. Pro, A. Ferri, A. Gola, N. Serra, A. Tarolli, N. Zorzi, and C. Piemonte, "New development of Near-UV SiPMs at FBK," IEEE Trans. Nucl. Sci., vol. 60, no. 3, pp. 2247-2253, Jun. 2013.

[13] A. Gulinatti, P. Maccagnani, I. Rech, M. Ghioni, and S. Cova, "35 ps time resolution at room temperature with large area single photon avalanche diodes," IEEE Electron. Lett., vol. 41, no. 5, pp. 272-274, Mar. 2005.

[14] S. Cova, A. Lacaita, M. Ghioni, G. Ripamonti, and T. A. Louis, "20 ps timing resolution with single photon avalanche diodes," Rev. Sci. Intrum. vol. 60, no. 6, pp. 1104-1110, 1989.

[15] M. Assanelli, A. Ingargiola, I. Rech, A. Gulinatti, and M. Ghioni, "Photontiming jitter dependence on injection position in single-photon avalanche diodes," IEEE J. Quantum Electron., vol. 47, no. 2, pp. 151-159, Feb. 2011.

[16] S. Seifert, H. T. van Dam, J. Huizenga, R. Vinke, P. Dendooven, H. Löhner, and D. R. Schaart, "Simulation of silicon photomultiplier signals," IEEE Trans. Nucl. Sci., vol. 56, no. 6, pp. 3726-3733, Dec. 2009.

[17] A. Gola, C. Piemonte, and A. Tarolli, "Analog circuit for timing measurements with large area SiPM coupled to LYSO crystals," in Proc. IEEE Nucl. Sci. Symp. Med. Imag. Conf., 2011, pp. 725-731.

[18] S. Seifert, R.Vinke, H. T. van Dam, H. Löhner, P. Dendooven, F. J. Beekman, and D. R. Schaart, "Ultra precise timing with SiPM-based TOF PET scintillation detectors," in Proc. Nucl. Sci. Symp. Conf. Rec., 2009, pp. 2329-2333.

[19] C. Piemonte, A. Ferri, A. Gola, A. Picciotto, T. Pro, N. Serra, A. Tarolli, and N. Zorzi, "Development of an automatic procedure for the characterization of silicon photomultipliers," in Proc. IEEE Nucl. Sci. Symp. Med. Imag. Conf. Rec., 2012, pp. 428-432.

[20] A. Lacaita, S. Cova, M. Ghioni, and F. Zappa, "Single-photon avalanche diode with ultrafast pulse response free from slow tails," IEEE Electron Device Lett., vol. 14, no. 7, pp. 360-362, Jul. 1993.
Fabio Acerbi (S'12-M'13) was born in Carpi, Italy, in 1984. He received the Bachelor's degree in electronics engineering in 2006, the Master's degree in 2008, and the Ph.D. degree in information technology in 2012.

From 2009 to 2013, he was involved in the development and characterization of new InGaAs/InP single-photon avalanche diodes. He is currently working on the development of SiPM and the related readout electronics.

Massimo Cazzanelli was born in 1972. He graduated in physics at the University of Trento, Trento, Italy, in 1996, he received the M.Sc. degree in pulsed laser deposition of GaN thin films from Trinity College Dublin and the Ph.D. degree in physics in 2003. He was a Research Assistant at the University of Geneva. In 2000, he started working at the University of Trento. His research interests include integrated nonlinear optics.

Alessandro Ferri was born in Bologna, Italy, in 1986. He received the Master's degree in physics from the University of Bologna, Bologna, in 2011.

He is currently a Junior Researcher. His current research interests include the development and characterization of silicon photomultipliers.

Alberto Gola was born in Milano, Italy, in 1978. He graduated in electronic engineering and he received the Ph.D. in information technology engineering from Politecnico of Milano, Milano, Italy.

He worked on silicon drift detectors and related optimal filtering and electronics. Since 2010, he has been working on development of SiPM and the related electronics.

Lorenzo Pavesi (M'08-SM'11) was born in 1961. He received the Ph.D. degree in physics in 1990. He is the Professor of experimental physics, Director of the Nanoscience Laboratory, and Head of the Department of Physics, University of Trento, Trento, Italy. He founded the research activity in semiconductor optoelectronics at the University of Trento. He was the first President and Founder of the IEEE Italian chapter on Nanotechnology. During the last years, his research interests include silicon-based photonics.

Nicola Zorzi was born in Milan, Italy, in 1961. He received the "Laurea" (M.S.) degree in physics in 1987. He joined Fondazione Bruno Kessler, Trento, Italy, in 1988.

He was involved in the development of radiation silicon detectors for highenergy physics, and space and biomedical applications. He contributed to the production of microstrip detectors for AMS and ALICE. His current research interests include development of silicon photomultipliers, drift detectors, and 3-D detectors.

Claudio Piemonte was born in Udine, Italy, in 1972. He received the "Laurea" degree (M.S.) in 1997.

From 1999 to 2002, he was with the National Institute for Nuclear Research, Trieste, Italy. In 2002, he joined Fondazione Bruno Kessler (FBK), Trento, Italy. His current research interests include the development of silicon radiation detectors, and low-level light sensors for high-energy physics experiments and medical applications. Since 2008, he has been coordinating the R\&D activity on these topics within FBK. 